International Journal of Electronics and Communication Engineering and Technology (IJECET) Volume 10, Issue 3, May-June 2019, pp. 19-32, Article ID: IJECET_10_03_004 Available online at http://iaeme.com/Home/issue/IJECET?Volume=10\&Issue=3

ISSN Print: 0976-6464 and ISSN Online: 0976-6472

(C) IAEME Publication

\title{
SAMCAR:Spectrum Aware Multi-Coefficient based shortest Anypath Routing in Cognitive Radio Networks
}

\author{
Shyleshchandra Gudihatti K N ${ }^{1}$, S H Manjula ${ }^{1}$, and Venugopal K R ${ }^{2}$ \\ ${ }^{1}$ University Visvesvaraya College of Engineering, Bangalore University, Bengaluru, India \\ Contact:shyleshchandra444@gmail.com,shmanjula@gmail.com \\ ${ }^{2}$ Bangalore University, Bengaluru, India
}

\begin{abstract}
Wireless communication technology has grown tremendously with an advancement of better communication quality. The communication quality depends on the available spectrum bands but due to increased demand, the spectrum scarcity has become a challenging issue. Besides, insignificant spectrum utilization in a wireless network degrades the performance issues. Recently dynamic spectrum management is proposed as a recommended solution by Federeal Communication Commission (FCC) which can enhance the spectrum management. Owing to this spectrum management, Cognitive Radio networks converging to offer possible solution. Accordingly, in this paper, we look at Cognitive Capacity Harvesting network and developed a mult-hop cognitive radio network routing protocol. The proposed routing protocol Multi-Coefficient based Shortest Anypath Routing (MCSAR) is devised based on the link quality, energy consumption and trust values for identifying the next-hop. Additionally, we incorporate the improved Bellman-Ford algorithm for further optimization of the communication. Generally, anypath routing provides two optimal paths in which selection of one optimal path becomes a challenging issue. In order to overcome this issue, we propose priority weight computation where distance from current node to destination node and its trust factors are considered for forwarding node selection. The performance of proposed approach is compared against conventional/existing anypath routing schemes. The extensive simulation result obtained ensures better efficiency in terms of packet delivery ratio, overhead of communication and computation, end-to-end delay, and throughput.
\end{abstract}

\section{Index Terms}

Anypath routing, Cognitive Radio Networks, Cognitive Capacitive Harvesting, Multi-Coefficient Shortest Anypath Routing, Routing Protocol.

\section{INTRODUCTION}

Recently, the wireless communication industries have faced a dramatic growth in various real-time application scenarios such as mobile health, online gaming, mobile social networks and so on. Nowadays, human, industries and other various systems are widely adopting the wireless services for everyday's requirement which leads to the increased demand of wireless communication radio spectrum. Consequently, the spectrum supply and availability plays a significant role in the wireless communication which can effect Quality of Experience $(\mathrm{QoE})$ and Quality of Service $(\mathrm{QoS})$ for end-users. Alternatively, Federal Communication Commission (FCC) has revealed the facts about scarcity of available spectrum to facilitate the promising high bandwidth communication. In addition, a study presented by FCC concluded that different licensed bands are not utilized effectively.

Accordingly, the better utilization of available spectrum can improve the communication performance. Based on these assumptions, Federeal Communication Commission (FCC) has suggested a new methodology for better utilization of spectrum by dynamically allocating the spectrum [1]. Thus, a new promising technique is developed to implement this concept is called as Cognitive Radio (CR) with the help of Cognitive Radio Networks (CRNs). This approach is able to efficiently sense the available spectrum and helps for better utilization. In Cognitive Radio networks, licensed users PU (Primary Users) and unlicensed users SU (Secondary Users) are two main components. The Secondary users utilize the available spectrum while safe guarding the Primary Users communication i.e., SUs can sense the spectrum without causing any interference to the PU. It is really demanding to address the issue for developing an effective routing protocol to calculate the optimal anypath in CRNs. In view of searching space evolves exponentially fast as the total number of applicable channels and the volume of the network grows. In general, basic concept of cognitive radio is simple and it can significantly improve the communication performance. However, due to increasing complexities and design variations the conventional cognitive radios pose several challenges such as analyzing the time-varying channel applicability which causes design complexity to the Medium Access 
Control (MAC) layer. Similarly, another critical issue is faced by SUs is that how to select the channel for transmitting or recieving the packets without inducing the communication between Primary Users (PU) [2].

To address the above said problems, in this paper, we present a novel Multi-Coefficient based Shortest Anypath Routing (MCSAR) for multi-hop CRNs. We deploy newly proposed network architecture for CRNs, named Cognitive Capacity Harvesting networks (CCHs) [3] [4][5], as a model to demonstrate our architecture. CCH includes Secondary Service Provider (SSP), Base Stations (BSs), Cognitive Radio routers (CR routers), and secondary users (SUs). SSP is the controller and administrator of $\mathrm{CCH}$, which yields spectrum resource and divides among the network. The $\mathrm{CR}$ routers are rigged with cognitive radios, which accomplish spectrum sensing, aggregate request from SUs, and communicate their information around over applicable licensed spectrum. SUs involve wireless equipments deploy conventional wireless access mechanism, and also covers wireless gadgets that are highly equipped with advanced cognitive radios for transmitting data.

The efficient routing protocol in CRN can provide the information about spectrum uncertainty and spectum-awareness in CRN. Moreover, it can provide reliable communication in unreliable communication environments. Particularly, in this paper our main objective is to study about recent routing protocols in CRN and developing a novel approach to overcome the drawbacks of existing protocols. In [6], authors discussed that contineous spectrum availability is rare. Therefore, spectrum aggregation approach can be helpful for efficient communication. Thus, Spectum Aggregation-based Cooperative Routing Protocol (SACRP) is introduced to mitigate the spectrum scarcity issues. In [7], routing protocol for multi-hop CRN is developed using a cooperative scheme which avoids interference, and improves the communication quality. Conventional approaches in CRN management consider only half-duplex transmission where as recent advancements have lead towards the full-duplex communication in CRN. In this type of networks, channel assignment and spectrum allocation is considered as a challenging task. Therefore, a new optimization framework is developed in [8].

Recently, zikria et al., [9] presented a novel approach for channel assignment and spectrum management for CRNs. They concluded that the opportunistic routing based schemes can significantly enhance the efficiency of the system. Based on this assumption of opportunistic routing protocol, anypath routing scheme is incorporated in CRN communication for spectrum management [10]. Liu et al., [11][12][13] exploits the broadcasting nature of wireless medium and present an opportunistic routing method. They suggested a cognitive communication throughput metric and introduce heuristic procedure to compute routing avenues. According to the conventional routing approaches, routing table management becomes a challenging task, thus opportunistic relay forwarding schemes are also developed [14]. However, the dynamic spectrum management is still considered as a challenging task in [10]. Finally concluded that Multi-Coefficient based Shortest Anypath Routing (MCSAR)can improve the performance efficiently utilizing the spectrum. Henceforth, we focus on spectrum management in cognitive radio networks.

\section{A. Problem Definition and Motivation}

Cognitive Radio networks are designed as a promising technique to advance the spectrum usage for communication quality and coverage for real-time wireless communications. Conventional schemes of CR are focused on the static spectrum management but due to increased demand of communication, the spectrum scarcity has become a challenging research issue. In order to overcome this issue, FCC has suggested dynamic spectrum access approach which can be implemented using efficient routing protocol. Several approaches have been introduced to overcome lack of issues which arise from the available spectrum and improper utilization of spectrum. A recent study presented in [10] concludes that anypath routing can improve the spectrum management in CRN.

\section{B. Work Contribution}

In this paper, we carry spectrum management in cognitive radio networks.

- Cognitive Capacity Harvesting $(\mathrm{CCH})$ network modelling for spectrum management

- Implementing the anypath routing for CRN.

- The existing approach of anypath routing considers link quality and energy consumption parameters for next-hop selection where as proposed approach incorporates trust value and geographical distance from current source nodes to destination node which makes anypath routing has multi-coefficient anypath routing.

- Comparative analysis to validate and show the performance improvement using proposed approach.

\section{Organization :}

The remainder of this paper is systematized as follows. In brief we study related work in section II. In section III, we summerize the system architecture design of $\mathrm{CCH}$, propose system model, illustrate the problems to be investigated and present the advanced routing schedule detail. We evaluate the advanced scheme and compare the simulation results in section IV. Finally concluding remarks presented in section V. 


\section{LiterATURE SURVEY}

In this section, we introduce a complete summary about recent techniques for spectrum sensing in the cognitive radio networks, Cognitive Capacity Harvesting Netwoks.

In the sphere of cognitive radio networks, game theory based approaches show a progressive improvement in routing due to cooperation between nodes and coalition formation which enhances the communication capacity in the network. Based on this assumptions, Guanet et al., [15] developed a game theory based approaches to improve the throughput of PU which leads towards increasing the accessing probability of licensed spectrum. This approach of spectrum sensing using game theory is also adopted for the dynamic spectrum sensing. In this context, Liu et al., [16] developed dynamic spectrum sensing approach for cognitive radio network which allows $5 \mathrm{G}$ wireless communication. This work mainly focuses on the two objectives particularly Interference cancellation among SUs and spectrum leasing. However, this work also shows that the existing techniques of interference cancellation are generalized for addressing the issue of SUs and PUs but mutual interference cancellation can be breakthrough for further enhancement in this area a game thoery based approach is developed in [16]. Fang et al., [17] focused on the trust based game model to establish energy efficient and secure communication in the cognitive radio network. Moreover, a distributed learnig approach is also applied which helps for selecting the relay node. The learnig approach uses automata technique which achieves Stackelberg equilibrium in the formulated game. Recently, Wang et al., [18] presented spectrum aware routing in multi-hop, multi-channel cognitive radio networks, which specifically focused on the blocking of mixed attack in the cognitive radio network. In this paper, a no-cooperative game is formed where Markov decision process is applied to decompose the non-cooperative game into multiple series of games and each game is realized with the distributed strategy allows by obtaining the equilibrium stages. Furthermore, trust based method is incorporated to achieve the security from different types of attacks. shylesh et al.,[19] studied the review on spectrum management techniques which supports sensing, deciding and allocation. This analyzes the energy efficient routing protocols with several metrics to route the available information from source to destination by optimally reducing the energy consumption. Finally describes the security issues in cognitive radio network and solutions to achieve the security for different types of attacks. On the other hand, energy and spectrum aware routing approaches also have gained huge attraction from research community. In this paper, energy aware and efficient spectrum sensing approaches play important role to enhance the overall performance of the system. Singh et al., [20] addressed the issue of spectrum scarcity for increasing the real time wireless multimedia communication. Authors studied that achieving the efficient routing protocol model for the dynamic environments in cognitive radio networks is a challenging task. In general, the conventional routing technique based on the shortest path compuation were link, and quality is ignored thereby the probability of poor communcation increases. However, the purpose of this shortest path selection appoach is to identify a path between the source and destination nodes. However, various other researchers used shortest path routing but the shortest path routing techniques fails to perform for multichannel algorithms in cognitive radio networks. Thus, authors presented cognitive radio routing protocol technique which introduces Smart Spectrum Selection (SSS) and Succeeding Hop Selection (SHS). These methods are used for selecting the relay node in the single process so communication overhead can be reduced. Ji et al., [21] also discussed about the routing issues in cognitive radio networks. The conventional methods for CRNs does not include the spectrum and resource dynamic due to increased cost of implemenation. Accordingly, in this paper, efficient routing approaches is developed to reduce the energy consumption and latency. This approach is divided into three stages where first of all, spectrum-aware semi-structure routing model is developed which helps to control the power consumption. In order to enhance the routing performance, the forwarding and routing zones are considered based on these zones, the dynamics of spectrum availability is obtained which helps to improve the global routing performance. This approach achieves the low power consumption by utilizing the lowest allowed working power. Moreover, a framework is introduced later which includes the latency and energy consumption model and resolves the energy related issues. In the case of routing in wireless networks, Dubois-Ferrin et al., [22] introduced an efficient approach for routing which can provide promising solution for the shortest path routing. This approach is called as anypath routing which achieves better routing performance with the help of optimal shortest path. Based on the advantages of anypath routing several approaches have been developed to upgrade the performance of cognitive radio networks. Chao et al., [23] discussed about the advantages of cognitive radio over conventional sensor networks. However, Primary Users occupancy generates several challenges to develop the routing model in cognitive radio networks. In the future, authors focused on the PUs occupancy and multi-channel rendezvous issues in cognitive radio networks. In order to overcome this issue, anypath routing is developed which helps to improve the overall performance of CRAHN's. Motivated by the advantages of anypath routing, Wang et al., [10] presented anypath routing based model for multi-hop cognitive radio networks and developed a novel routing approach called as spectrum-aware anypath routing (SAAR). We develop a new multi-coefficient routing metric, which considers both channel uncertainty and unreliability of wireless medium. On account of this routing metric, we also design an efficient MCSAR (Multi-Coefficient Shortest Anypath Routing) algorithm to discover the priorities of vacant channels and analogous forwarding sets at each node. This approach has significant routing performance for the unreliable transmission characteristics. In this paper, we present Multi-Coefficient based shortest anypath routing. 


\section{PRoposed Model}

In this section, we study detailed discussion of anypath routing introduced in [22], further motivation which helps to carry this work, finally formulation of the problem and present the proposed solution which are defined.

\section{A. Overview of Anypath Routing}

In conventional wireless network based communication, a communicating node broadcasts a packet to the single next hop. This scheme is applicable for the environment where less data traffic is experienced. Furthermore, due to the recent technological growth in telecommunication industry has led towards the generation of heavy traffic by using cognitive radio based communication standards. In this type of scenario, the data traffic is heavy and due to frequent communication, the probability of packet drop is higher. According to the conventional approaches, if the routed packet is dropped then it needs to retransmit the packet which can cause the network overhead. Threrefore, traditional single next-hop packet forwarding schemes cannot be utilized for these scenarios.

On the other hand, according to the anypath routing approach, the packets are forwarded to the multiple next hop concurrently. If the packet transmission to one node declines, then the other node can receive this packet and can forward it. This approach is widely adopted due to next hop transmission because if the packet is dropped by one node then it is collected by another next-hop and forwarded further which helps to improve the communication performance. From the concept of opportunistic routing, it is generalized that more number of neighbouring node can effectively increase the probability that at least one node from the all neighbours can receive the packet. We describe these set of nodes as forwarding set and denote it using $J$. In this approach, the node $v$ is known as forwarder node of node $u$ and $u$ is known as forwarding node of $v$. However, during communication process, the neighbouring node may receive the same type of packets. Henceforth, we define the probability of node to forward the packet which depends on the distance and cost computed from the current node to destination node. With the help of this probability, the node with the higher probability can forward the packet where as other nodes forwarding is suppressed. This process of packet broadcasting is repeated until packet broadcasting threshold is achieved or the packet reaches to the destination node.

According to [22], the anypath routing follow directed acyclic graph where each node (source) is a successor node of the source and each node (sink) is predecessor of the destination node. Here, we employ Fig. 1 to demonstrate how an anypath takes place. Moreover, due to the acyclic nature potential path is selected and no packet will pass over the same forwarder node more than once. In the given Fig. 1 shows working principle of anypath routing where green dotted lines depicts the possible path for a packet to travel from source node $s$ to destination node $t$. A general network model for anypath network routing is represented in figure where we have considered 7 nodes in this scenario. Based on the general scenario of routing, we obtain two paths to reach to destination including multiple forwarding sets. These two paths are described in Fig.(1)b and Fig.(1)c. Let us consider that $P_{v}$ denotes the anypath from source node $s$, destination node $t, u$ denotes the any node in the path. We consider this model and apply it for Cognitive Capacity Harvesting Networks (CCHN)

\section{B. System Architecture and System Model}

In this section, we introduce the system architecture and modeling of $\mathrm{CCH}$ networks. The basic architecture and system model is adopted from [10]. A system architecture of CCH is depicted in Fig. 2 which contains four type of network objects such as Secondary Service Provider (SSP), Base Stations, Secondary Users and Cognitive Radio routers.

It is assumed that the basic band and other components of networks can assume the available band to specify the reliable communication. With the help of CR routers and BSs, SSP harvests the available spectrum resources and allocates the available resources to accomplish the demand which is used for providing the communication services to the Secondary Users (SUs). In other words, the SSP acts as a manager, BS acts as a gateway which is used for connecting to the internet and other service. CR routers \& BSs acts as access points that helps to SUs to acquire the $\mathrm{CCH}$ where SUs can be any kind of wireless device. These wireless devices employs any accessing technique such as Laptops uses WiFi technique, cellular mobile phones uses GSM/GPRS/3G/4G technique. As discussed in [8], the achievement of $\mathrm{CCH}$ rely on the routing protocol consequently development of a reliable routing protocol is highly desirable.

Let us consider that $N_{B}$ number of Base stations and $N_{R}$ number of CR routers are harnessed with multiple cognitive radio in the considered $\mathrm{CCH}$ and all nodes are denoted by $N=N_{B}+N_{R}$. As discussed previously that BSs and CR routers cooperate each other which helps Secondary Service Provider (SSP) to acquire the statistical information about each channel. In this paper, we model the $\mathrm{CCH}$ network in the form of hypergraph as $\mathcal{G}=(\mathcal{V}, \mathcal{E})$ here $\mathcal{V}$ denotes the set of CR routers and BSs. $\mathcal{E}$ denotes the set of hyperlinks. According to this model, each edge $m$ between $(v, u) \in \mathcal{E}$ has a Packet Delivery Probability as $p_{v, u}^{m}$. As discussed earlier $J$ denotes the forwarding set of node $v$ and a Hyperlink Delivery Probability (HDP) $\mathcal{P}_{v, J}^{m}$ determines the probability where a packet transmitted by node $v$ is received by atleast one of the nodes in set $J$. During communcation phase, the packet drop can occur at receiver thus the HDP can be computed as:

$$
\mathcal{P}_{v, J}^{m}=1-\prod_{j \in J_{v}^{m}}^{m}\left(1-p_{v, j}^{m}\right)
$$




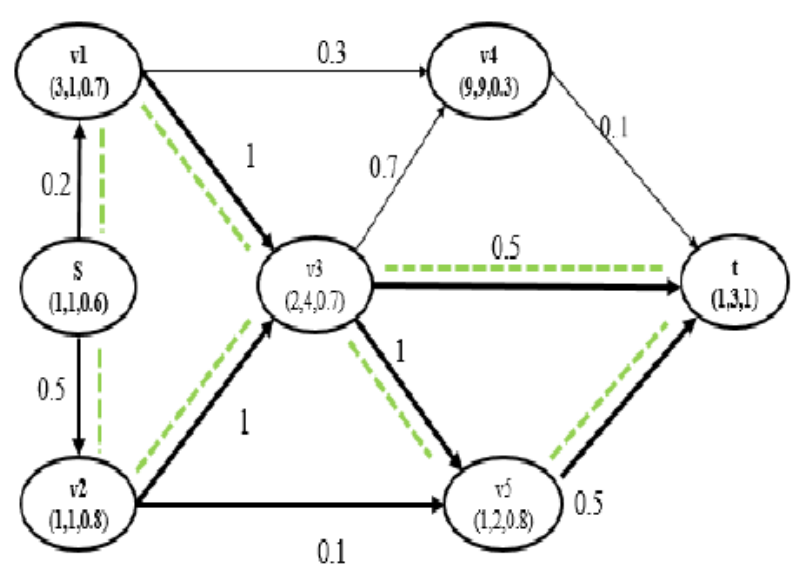

(a) Anypath representation for $P_{s}$

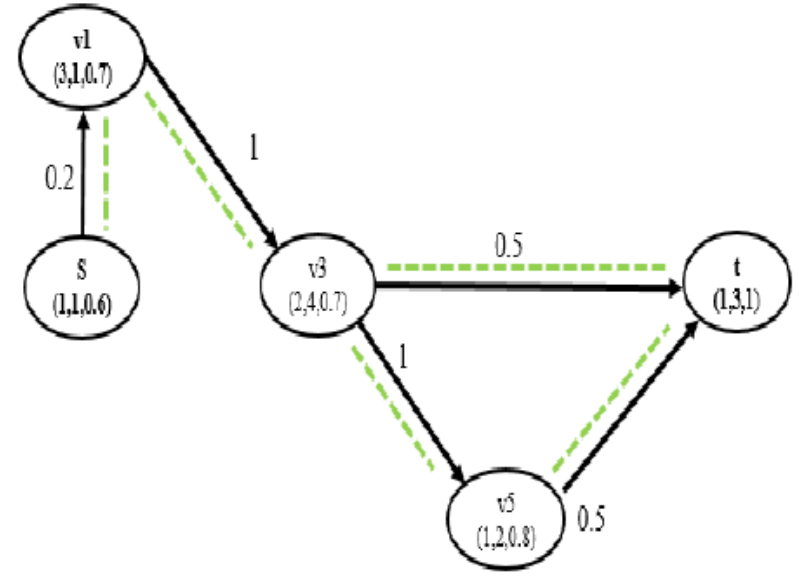

(b) Anypath representation from $s$ to $t$ (Path 1)

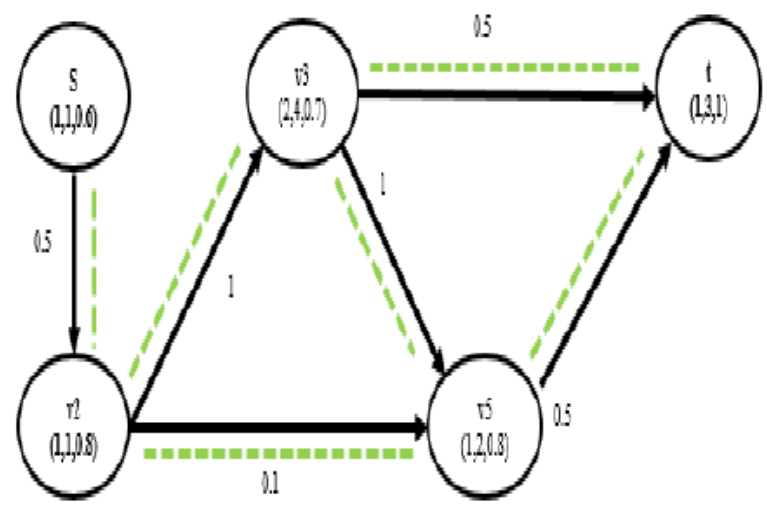

(c) Anypath representation from $s$ to $t$ (Path 2)

Fig. 1: Anypath and Sub-path Representation

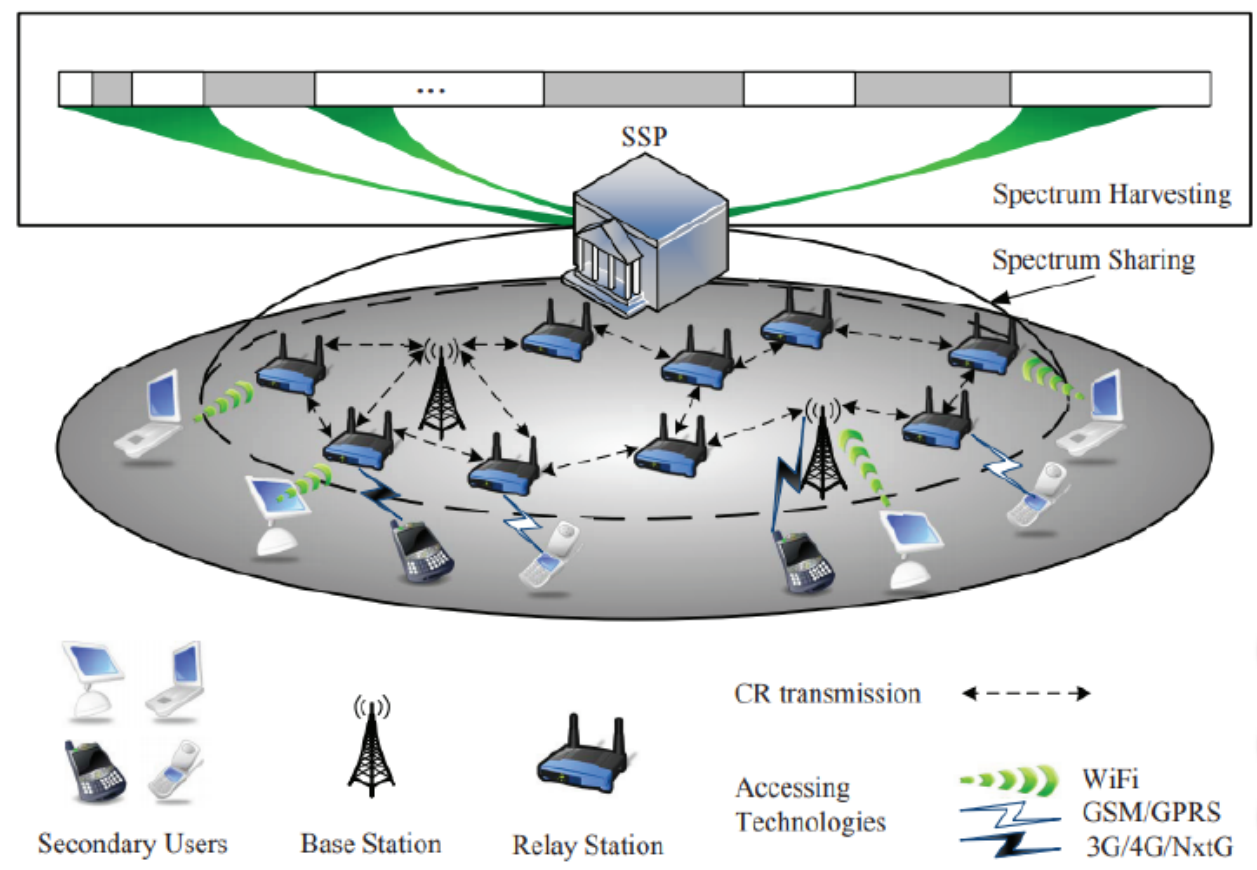

Fig. 2: Basic system model of $\mathrm{CCH}$ network 
$j$ is the forwarding set of $J$ and $m$ denotes the available channels. Let us consider a set of nodes as $J=\left\{v_{1}, v_{2}\right\}$ which are the forwarding set of node $s$ according to the Fig. 1 . however, the HDP can be computed as $P(s, J)=1-(1-0.2)(1-0.5)=0.6$ by using equation (1).

Generally, the Medium Access Control (MAC) inform the network layer regularly to restore the average energy conumption, transmission time, delivery probability and other related information. Each node shares this information to neighbouring nodes with control packets. Furthermore, the MAC must take care of schedule links, interference, collision and restore from packet loss. The analysis of the MAC, however, are abstracted from the routing layer. The design of MAC is not studied in this paper.

Similarly, each node is associated with the $K$ weights vertices denoted as $w_{k}(v), 1 \leq k \leq K$ where $K$ denotes the integer value of the total number of considered weights. In our case, $K=1$ represents the average time necessary by a node $v$ to complete the transmission expressed as $w_{1}(v), K=2$ denotes the energy consumed during the data transmission expressed as $w_{2}(v)$ and $K=3$ denotes the trust value $(T)$ for transmission expressed as $w_{3}(v)$. Based on the said considered parameters i.e, weights of vertex, delivery probability and trust value. We present a metric as Trusted Optimal Weight for Transmission (TOWTX), which can be formulated as:

$$
\operatorname{TOWTX} \varsigma_{k}(v, J)=\frac{w_{k}(v)}{p(v, J)}+T
$$

Where $k, 1 \leq k \leq K$, as mentioned earlier, in this work we have considered $\mathrm{K}=3$. Further as depicted in equation (1), the expected number of total packets forwarded from node $v$ to $J$ can be computed as: $\frac{1}{1-(1-0.2) \times(1-0.5)}=1.66$. Similarly, if $w_{1}(s)=2$, then the expected transmission time for a packet forwarded from node $v$ to $J$ can be given as $\frac{2}{1-(1-0.2) \times(1-0.5)}=3.33$. Consider the $H D P$ from node $v$ to forwarding set as $J\left(v, P_{s}\right)=j_{1}, j_{2}, j_{3}, \ldots j_{k}$, can be expressed as

$$
P\left(v, J\left(v, P_{s}\right)\right)=1-\Pi j_{\beta} \in J\left(v, P_{s}\right)\left(1-p\left(v, j_{\beta}\right)\right)
$$

Here, we define a coefficient for the forwarding set as $\psi\left(j_{\beta}, P_{s}\right)$ which can be expressed as:

$$
\psi\left(j_{\beta}, P_{s}\right)=\frac{p\left(v, j_{\beta}\right) \Pi_{q=1}^{\beta-1}\left(1-p\left(v, j_{q}\right)\right)}{1-\Pi_{j_{\beta} \in J\left(v, P_{s}\right)}\left(1-p\left(v, j_{\beta}\right)\right)} \forall j_{\beta} \in J\left(v, P_{s}\right)
$$

In equation (4), the numerator denotes the probability of successful packet transmission of any node from the forwarding set with the $\beta$ priority factor and the denominator is normalizing constant such as $\Pi_{j} \in J\left(v, P_{s}\right)\left(1-p\left(v, j_{\beta}\right)\right)=1$. Based on these parameters, $k^{\text {th }}$ anypath weight of forwarding set $J\left(v, P_{s}\right)$ along anypath $P_{s}$ can be calculated as:

$$
\mathbf{W}_{k}\left(J\left(v, P_{s}\right), P_{s}\right)=\underset{j_{\beta} \in J\left(v, P_{s}\right)}{\mathrm{X}} \psi\left(j_{\beta}, P_{s}\right) \mathbf{W}_{k}\left(j_{\beta}, P_{s}\right)
$$

With the help of equation (4) and (5), we compute the anypath weight for packet forwarding from node $v$ to destination $t$, which is characterized as:

$$
\mathbf{W}_{k}\left(v, P_{s}\right)=\frac{w_{k}(v)}{p\left(v, J\left(v, P_{s}\right)\right)}+\mathbf{W}_{k}\left(J\left(v, P_{s}\right), P_{s}\right)
$$

With the help of equation (4) and (5), we can rewrite as:

$$
\begin{aligned}
\mathbf{W}_{k}\left(v, P_{s}\right) & =\frac{w_{k}(v)}{p\left(v, J\left(v, P_{s}\right)\right)} \\
+ & \frac{\mathrm{P} \quad j_{\beta} \in J\left(v, P_{s}\right) \mathbf{W}_{k}\left(j_{\beta}, P_{s}\right) p\left(v, j_{\beta}\right) \Pi_{q=1}^{\beta-1}\left(1-p\left(v, j_{q}\right)\right)}{p\left(v, J\left(v, P_{s}\right)\right)}
\end{aligned}
$$

Where $k=1,2,3, . . K$. In this paper, we have considered $K=3 . w_{1}(v)$ denotes the average time required for transmission. $w_{2}(v)$ denotes average energy consumption requirement for every transmission, and $w_{3}(v)$ depicts the trust value. Similarly, the expected transmission time $\mathbf{W}_{3}\left(v, P_{s}\right)$ for transmitting data packet from node $v$ to $t$ along with anypath $P_{s}$. In this process, the trust can be computed based on the successful cooperation between nodes, this can be presented as:

$$
T 1=\frac{S}{S+F}
$$

Where $S$ denotes successful co-operations, $F$ denotes failed co-operations. However, in some cases we may obtain the similar trust value thus we incorporate a distance metric which is given as:

$$
T 2=1-\frac{d_{i}}{D}
$$

Where $d_{i}$ describe the Euclidean distance among neighbor node $j$, and the base station, and $D=\mathrm{P}_{i}^{N} d_{i}$, where $N$ denotes the next-hops. Finaly, the trust value is expressed as $T=T 1+T 2$. 


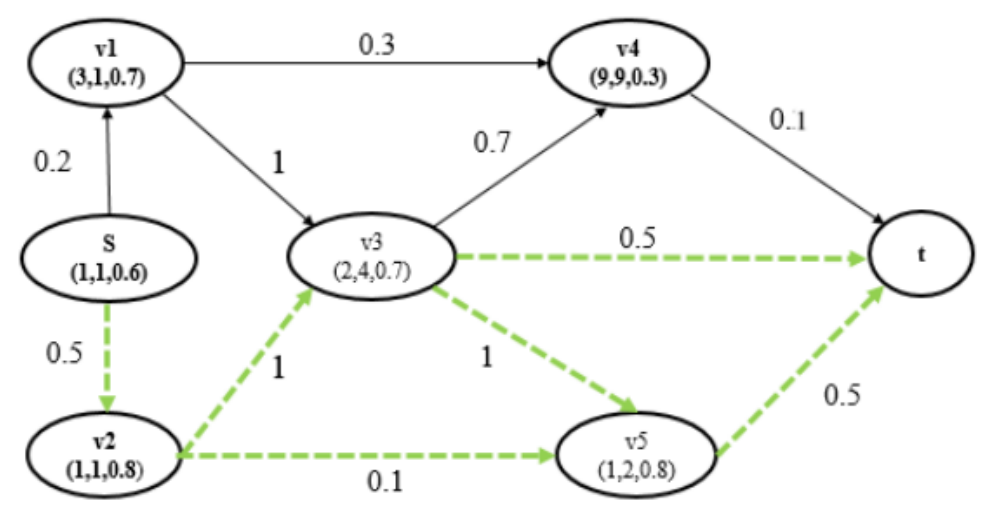

Fig. 3: Anypath Weight Computation

TABLE I: Optimal Anypath and Metric Weight Calculation

\begin{tabular}{|c|c|c|c|c|c|c|c|}
\hline Stage & $\begin{array}{c}\text { Considered } \\
\text { node }\end{array}$ & $\begin{array}{c}\text { corresponding } \\
\text { Coefficients } \\
\end{array}$ & $\begin{array}{c}\text { Corresponding } \\
\text { forwarding node }\end{array}$ & forwarding node & $\begin{array}{c}\text { Link } \\
\text { Probability }\end{array}$ & $\begin{array}{c}\text { Anypath } \\
\text { Weight }\end{array}$ & $\begin{array}{l}\text { Metric } \\
\text { weights }\end{array}$ \\
\hline 1. & $t$ & $(1,3)$ & NA & NA & NA & $\mathbf{W}_{1}\left(t, P_{s}\right)=0$ & $(0,0)$ \\
\hline 2. & $v 5$ & $(1,2)$ & $t$ & $(1,3)$ & 0.5 & $\mathbf{W}_{1}\left(v 5, P_{s}\right)=2$ & $((2,4), 4)$ \\
\hline 3. & $v 3$ & $(2,4)$ & $\mathrm{v} 5, t$ & $(1,2),(1,3)$ & $1,0.5$ & $\mathbf{W}_{1}\left(v 3, P_{s}\right)=3$ & $((3,6), 6)$ \\
\hline 4. & $v 2$ & $(1,1)$ & $\mathrm{v} 3, t$ & $(1,1),(2,4)$ & 1 & $\mathbf{W}_{1}\left(v 2, P_{s}\right)=3.9$ & $((12,14), 14)$ \\
\hline 5. & $s$ & $(1,1)$ & $\mathrm{v} 1, \mathrm{v} 2$ & $(3,1),(1,1)$ & $0.2,0.5$ & $\mathbf{W}_{1}\left(s, P_{s}\right)=5.9$ & $((0,0), 0)$ \\
\hline 6. & $v 1$ & $(3,1)$ & $\mathrm{v} 4$ & $(9,9)$ & 0.3 & $\mathbf{W}_{1}\left(v 1, P_{s}\right)=6$ & $((6,7), 9)$ \\
\hline 7. & $v 4$ & $(9,9)$ & $t$ & $(1,3)$ & 0.1 & $\mathbf{W}_{1}\left(v 1, P_{s}\right)=90$ & $((90,90), 90)$ \\
\hline
\end{tabular}

Let us consider Fig. 3 to compute the anypath weights where the anypath $P_{s}$ is presented in dotted green lines which can be arranged as $s \rightarrow v_{2} \rightarrow v_{3} \rightarrow v_{5} \rightarrow t$. At this stage the anypath cost of destination node is 0 i.e, $\mathbf{W}_{1}\left(t, P_{s}\right)=0$. In this case, we have selected the path based on the trust factor i.e. the node $v_{1}$ has the trust factor as 0.7 whereas node $v_{2}$ 's trust factor is 0.8 . We consider node $v_{5}$ for further process and compute the anypath weight of $v_{5}$ as follows:

$$
\mathbf{W}_{1}\left(v_{5}, P_{s}\right)=\frac{w_{1}\left(v_{5}\right)+\mathbf{W}_{1}\left(t, P_{s}\right) \times p\left(v_{5}, t\right)}{p\left(v_{5}, t\right)}
$$

Based on equation (10) the anypath weight is obtained as $\frac{1+0 \times 0.5}{0.5}=2$ Similarly, the anypath weight from node $v_{3}$ to the destination $t$ via forwarding set $J\left(t, v_{5}\right)$ can be computed as:

$$
\begin{aligned}
& \mathbf{W}_{1}\left(v_{3}, P_{s}\right)=\frac{w_{1}\left(v_{3}\right)}{1-\left(1-p\left(v_{3}, t\right)\right)\left(1-p\left(v_{3}, v_{5}\right)\right)} \\
& \quad+\frac{p\left(v_{3}, t\right) \mathbf{W}_{1}\left(t, P_{s}\right)+\left(1-p\left(v_{3}, t\right)\right) \mathbf{W}_{1}\left(v_{5}, P_{s}\right) p\left(v_{3}, v_{5}\right)}{1-\left(1-p\left(v_{3}, t\right)\right)\left(1-p\left(v_{3}, v_{5}\right)\right)}
\end{aligned}
$$

With the help of Equation (11), the anypath weight can be of $v_{3}$ to $t$ via $v_{5}$ can be obtained as $\frac{2+0 \times 0.5+(1-0.5) \times 2 \times 1}{1-(1-0.5) \times(1-1)}$ $=3$

Similarly, remaining anypath weights from node $v_{2}$ and source node $s$ are also computed as $\mathbf{W}_{1}\left(v_{2}, P_{s}\right)=3.9$ and $\mathbf{W}_{1}\left(s, P_{s}\right)=5.9$ respectively.

In this paper, we define secondary anypath weight metric $\mu_{\max }(v)=\max _{1 \leq k \leq K} \frac{w_{k}(v)}{\mathbb{W}_{k}}$ where $\mathbb{W}_{k}$ represents $k^{\text {th }}$ Quality of Service $(\mathrm{QoS})$ constant from node $v$ to destination node $t$ along with the available anypath $P_{s}$, which is written as:

$$
\mathbf{W}_{1}\left(v, P_{s}\right)=\frac{\mu_{\max }(v)}{p\left(v, J\left(v, P_{s}\right)\right)}+\mathbf{W}_{\max }\left(J\left(v, P_{s}\right), P_{s}\right)
$$

Where $\mathbf{W}_{\max }\left(J\left(v, P_{s}\right), P_{s}\right)=\mathrm{P}_{j_{\beta} \in J\left(v, P_{s}\right)} \psi\left(j_{\beta}, P_{s}\right) \mathbf{W}_{k}\left(j_{\beta}, P_{s}\right)$

Here, $\mathbf{W}_{\max }\left(J\left(v, P_{s}\right), P_{s}\right)$ denotes the Secondary Anypath Weight (SAW) metric for the considered forwarding path set $J\left(v, P_{s}\right)$. This SAW metric is used for identifying the optimal anypath weight which helps to identify the optimal path. There 
can be two paths from source to the destination, one is computed using proposed approach thereby selection of one optimal path is a challenging task. The process of selecting the optimal path is discussed in this subsection $C$ where we consider the trust factor and minimal distance from the destination node based on their geographical locations respectively. Similarly by considering this process, we obtain the complete optimal path from source to destination node. The proposed approach is presented below given table. 1 where current node, corresponding link probability, forwarding set and obtained anypath weights are demonstrated.

Based on this approach, we present a complete method to calculate the optimal path from source node to destination node. This process carried out along with their corresponding weights, links and obtained distance is depicted in Fig. 3 which shows that the optimal path is obtained in 7 consecutive iterations. We begin our approach from the destination node where $t$ node is considered in the first iteration. In the second iteration, we consider the node $v_{5}$ where link labels indicates the data delivery probability and vertices shows the weights. In the subsequent iteration, anypath weights are computed via forwarding node $v_{5}$ to the destination node $t$, the $w_{1}$ of node is given as $2, w_{2}$ is given as 4 and the delivery probability between $v_{3}$ and $v_{4}$ is given as 1. Similarly, these iterations are processed until the source node is reached and the complete path is denoted as optimal path among source node $s$ and destination node $t$.

At the current stage, we have a hypergraph as $\mathcal{G}=(\mathcal{V}, \mathcal{E})$ where Packet Delivery Probability $(\mathrm{P})$, trust value for each node are present which can be used for routing the packets in $\mathrm{CCH}$. Here, our main aim is to find the optimal solution with least path cost, and energy consumption from source to destination node using anypath routing way. According to the previous discussion about the optimal path selection, we consider aforementioned $\mathrm{CCH}$ network and applied the anypath routing which provides the optimal shortest paths from source to destination node. Further in order to improve the performance of the system, we present an improved Bellman-Ford algorithm for computing the low-overhead and shortest path. However, Dijkastra's algorithm is widely adopted for shortest path computation but in this scenario, negative weight edges hypergraph based network architecture is presented which cannot be solved using Dijkastra's algorithm. According to this approach, each node maintains a distance value in such a way that is considered as an upper bound of the real-distance from node $s$ to $u$. The Bellman-Ford approach is adopted from [24].

After analyzing the given algorithm, we present an anypath weight computation process where $K=3$ as described in previous sections. During the initialization phase, all necessary parameters are assigned to their corresponding parameters such as $\mathcal{W}_{m}$ is assigned $\infty, W_{k}\left(v, P_{s}\right)$ is assigned as 0 . In the next phase, a new link is inserted to compute the anypath weights. Later, line 14-29 are used for computing the link quality matrix and update it iteratively. If the values of anypath weight are decreasing then the forwarding sets are updated. Similarly, this process is repeated until the shortest optimal path between source and destination is identified. The complete process is divided into three stages such as initialization, anypath weigh computation and optimal path selection.

However, the algorithm 1 provides optimal path because it generates two optimal anypath which are in the factor of 2 . In the given scenario we obtained two path values such as $\frac{5.9}{1}, \frac{8.8}{1}=8.8$ and $\frac{5.91}{1}, \frac{8.5}{1}=8.5$ correspondingly. The selection of optimal path becomes a crucial task in this scenario. Therefore, we introduce a new scheduling scheme for packet transmission as discussed in the next sub-section.

\section{Scheduling approach for optimal path selection}

In this subsection, we present a solution for selecting the one optimal path problem in anypath routing. According to this mechanism the nodes are assigned with priority weights and higher priority weights are considered as the next hop in the selected path $P_{v}$. During this period, the other neighbouring nodes will not transmit the packets until the priority weight of communicating node changes or its transmission duration expires. Once the transmission duration expires, the other nodes starts transmitting the packets. However, these scheduling approaches gradually leads to more waiting time and network overhead. In order to overcome this problem, we present a novel approach where each node is provided a weight priority and an optimal waiting time for node in the network.

In order to compute the weight priority of a node, we incorporate geographical distance computation for the node. This distance is computed between current node and destination node. Thus, the weight priority of a node can be calculated as:

$$
\mathcal{W}_{j}=\frac{\text { Dist }_{s, t}-\text { Dist }_{j . t}}{\text { TOWTX }_{j}^{2}}
$$

Where Dist $t_{s, t}$ represents the geographical distance among source and destination node, and Dist $_{j . t}$ represents the distance among forwarding node and destination node. Based on this assumption (13), we compute the high priority node with the trust factor to obtain the final anypath route. Hence, with this idea, we can achieve the one optimal path to transmit the packet from source to destination node.

After the execution of Algorithm. 1, we are able to discover the optimal anypath from each node to a particular destination set. By cooperating with the forwarding sets of each channel and their matching priorities along the anypath. For easy understanding, we consider an example to demonstrate the execution of MCSAR routing algorithm 1. The topology of a network and notations used are identical in Fig. 1. From the figures, we observerd the execution of MCSAR algorithm, each node discovers its optimal 


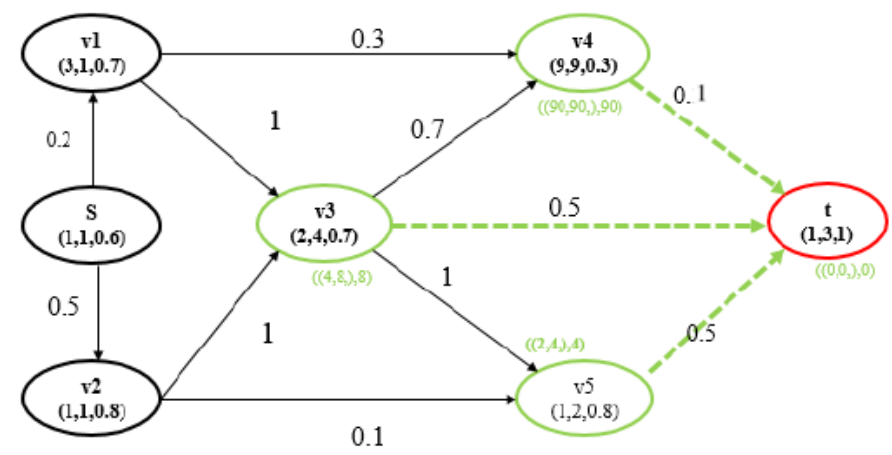

(a) t node scenario

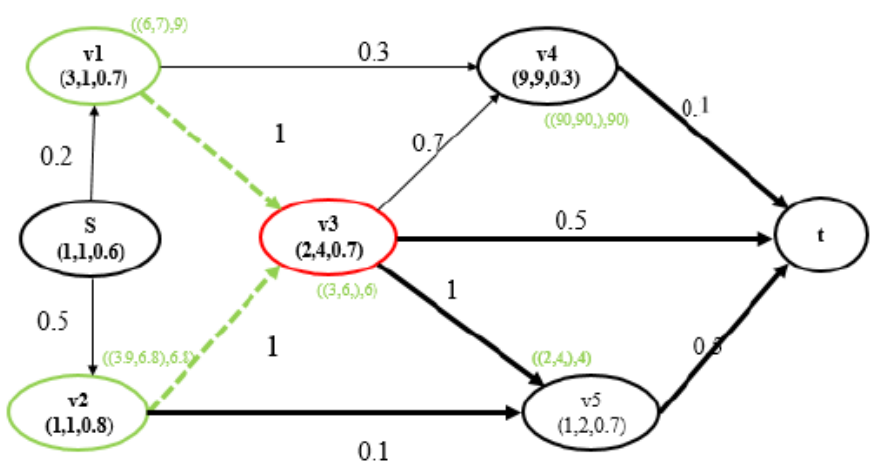

(c) v3 node scenario

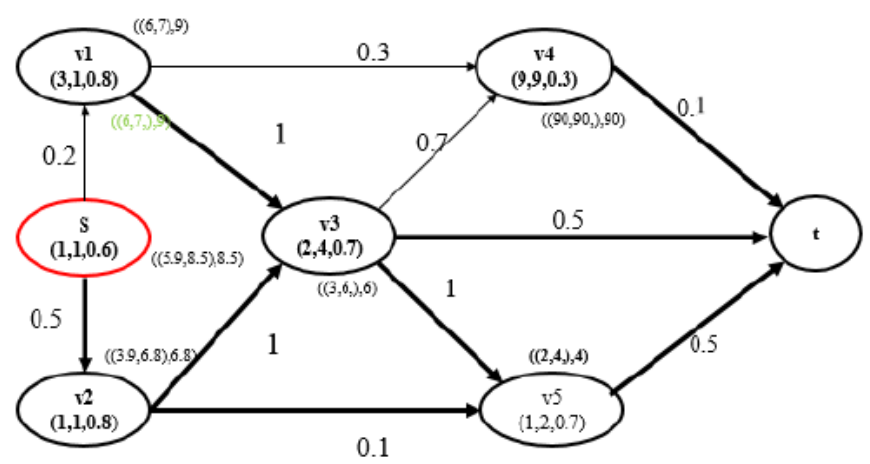

(e) s node scenario

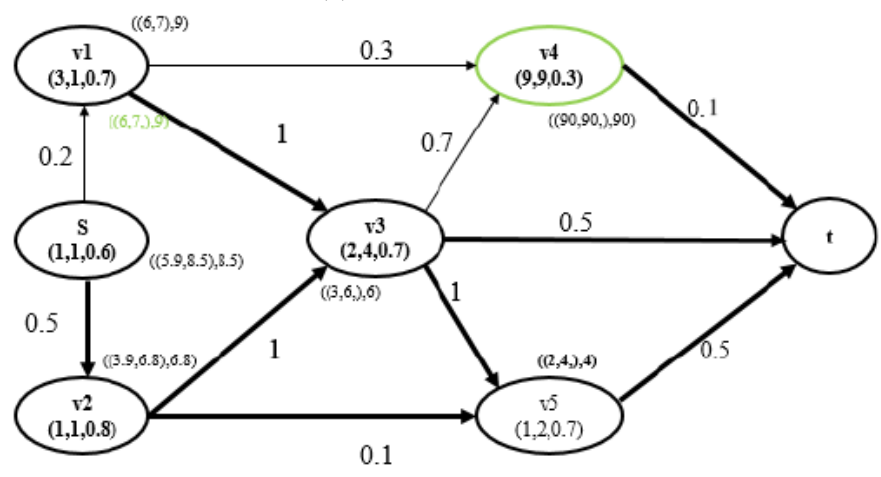

(g) v4 node scenario

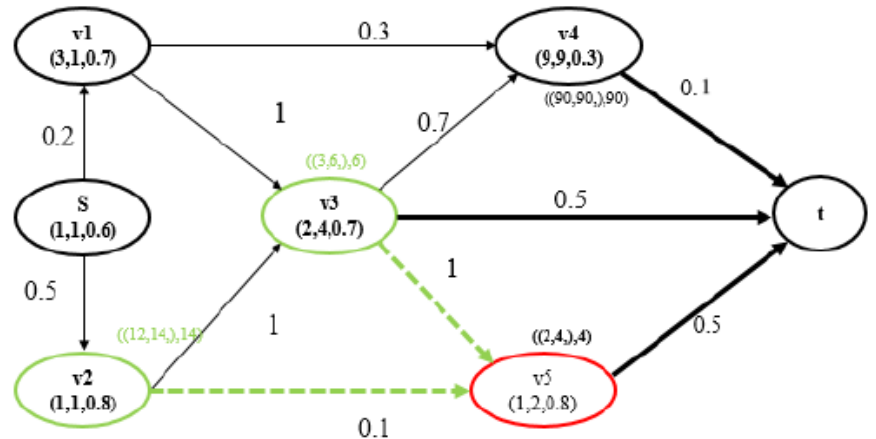

(b) v5 node scenario

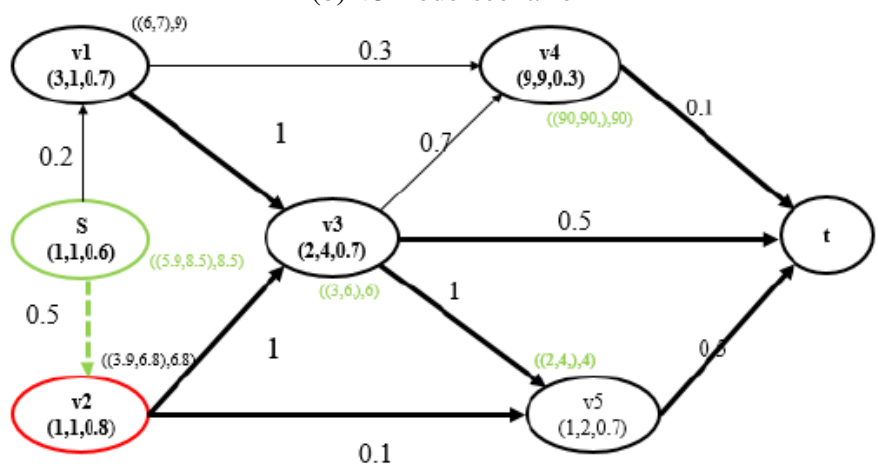

(d) v2 node scenario

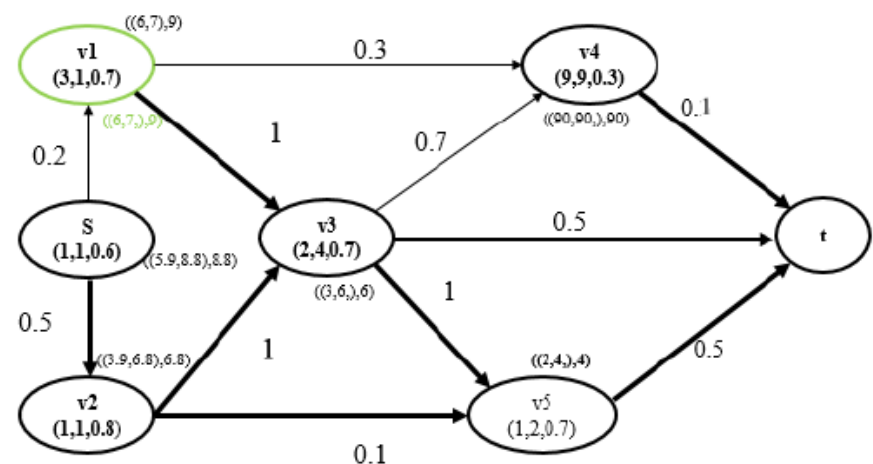

(f) v1 node scenario

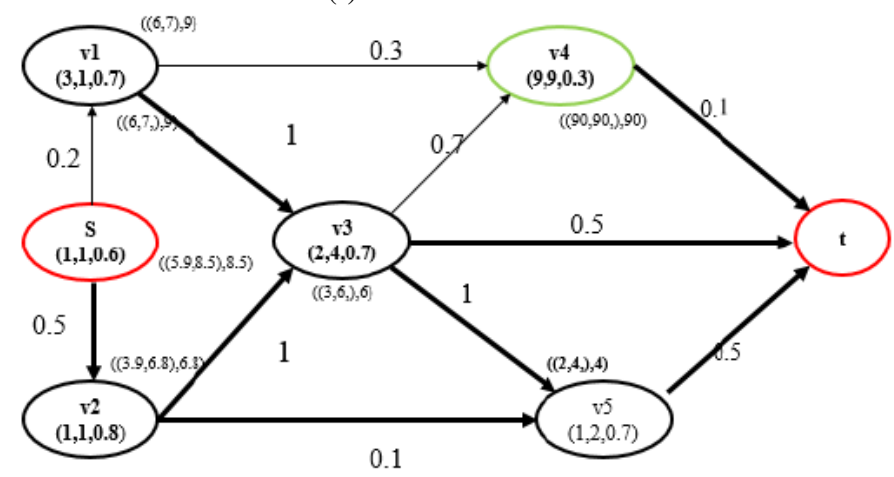

(h) Final optimal path

Fig. 4: Anypath Computation Process 


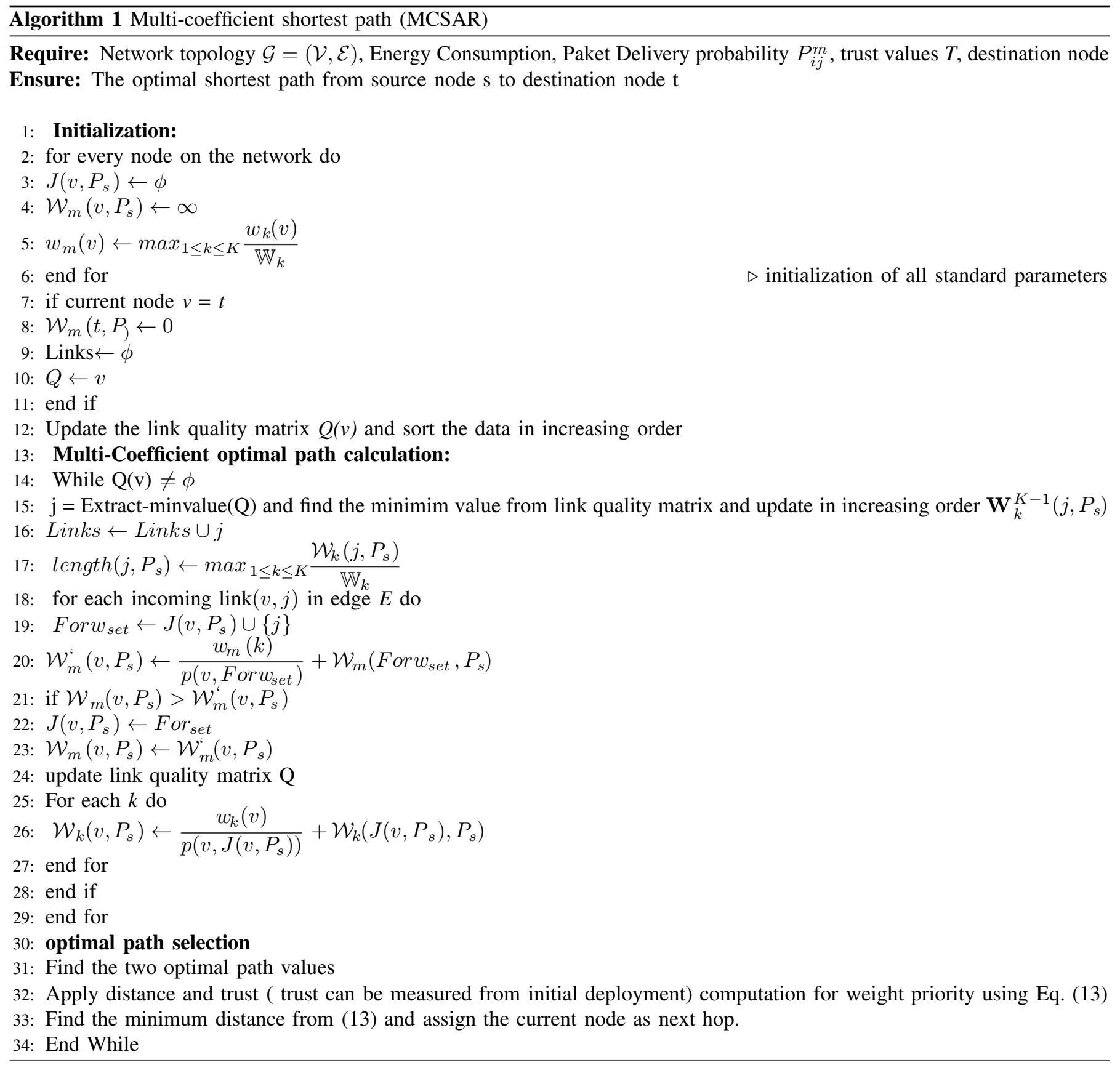

weighted metric anypath to 1 or 2 nodes in the destination node set. Meanwhile, we ascertained different nodes have dissimilar number of paths to the gateways. Consider an example anypath of node $v_{5}$ is a single path, while the anypath of $v_{3}$ consists of 5 paths, with 3 paths to $t$ and 2 paths to $s$. In consideration of MCSAR routing algorithm running by the SSP that has powerful computational power and time complexity is acceptable in this scenario. Further, we have proved the optimality of the advanced routing algorithm MCSAR. Also it inherits the characterstics of anypath routing algorithms for traditional wireless networks.

The proposed MCSAR can also be developed in a distributed passion, where every node maintains computing the anypath metrics purely on local information and mutually transfering knowledge with the neighbours till the anypath metric and optimal forwarding set are balanced. This approach is more appropriate for distributed cognitive radio networks like coginitive radio ad-hoc networks.

\section{RESUlTS AND Discussion}

In this section, we analyze the efficiency of the considered MCSAR method by conducting thorough simulations, and obtained values presents on packet delivery ratio (PDR), throughput, and end-to-end delay under different network setup like number of $\mathrm{CR}$ routers, channel conditions, and source data rates etc. In this simulation particularly we have considered $K=3$ constraints 
TABLE II: Simulation Parameters

\begin{tabular}{|l|l|l|}
\hline $\begin{array}{l}\text { SI } \\
\text { No }\end{array}$ & Simulation Parameters & Considered Value \\
\hline 1. & Network Area & $1000 \mathrm{~m} \times 1000 \mathrm{~m}$ \\
\hline 2. & Number of channels & 4 \\
\hline 3. & Number of PU per Channels & 16 \\
\hline 4. & Transmission Range of PU & $250 \mathrm{~m}$ \\
\hline 5. & Total No of BSs & 4 \\
\hline 6. & Total No of Routers & 150 \\
\hline 7. & Transmission Range of BS and CR & $120 \mathrm{~m}$ \\
\hline 8. & Data traffic type & constant bit rate \\
\hline 9. & Flow rate & $5 \mathrm{kbps}$ \\
\hline 10. & Packet size & $512 \mathrm{kbps}$ \\
\hline 11. & Channel availability probability & $0.7,0.3,0.6,0.8$ \\
\hline
\end{tabular}

where time, energy and trust values are studied to provide the optimal path from source to destination node. This simulation setup is conducted using Matlab software with windows operating system platform. Here, we assume a CCH network with a SSP, multiple PUs, BSs, and CR routers. The SSP is placed at the center of the networks, while all other network entities distributed rationally in a $1000 \mathrm{~m} \times 1000 \mathrm{~m}$ square region. There are 500 test cases conducted by considering the distribution of every nodes in $\mathrm{CCH}$ and the source-destination pair placed randomly in each test. A stable bit rate flow is cooperated with packet size of 512 Bytes and flow rate of $5 \mathrm{Kbps}$. The physical layer is designed by employing the log-normal shadow fading model. However, the MCSAR approach requires a MAC which helps anycast with priority, we adopt the anycast MAC [11], [25], [26] in our simulation. The network parameters required default shown in Table 2.

Here, we evaluate the achievement of various algorithms under different Primary User set up and setting channel availability probability to be $0.7,0.3,0.6$, and 0.8 , respectively. The obtained results of proposed approach is analyzed with the existing SAAR (Spectrum Aware Anypath Routing) [10] Unicast Routing (SAR-1) and Opportunistic Cognitive Routing (OCR) [11]. Particularly the proposed approach considers channel probability availability parameter and compute the enhancement in terms of packet delivery rate and End-to-End Delay are shown in Fig. 5 and Fig. 6. A little variation in channel availability identifies that the PU has regular activity, and assumed that the transmissions of CR router set up are more likely to be disturbed. Hence, the PDR is comparatively smaller and end-to-end delay is bit larger. Similarly the slight change in channel availability probability PDR will grow and end-to-end delay will diminish gradually. Here, channel availability plays important role to deliver the packet successfully to the destination node. If less channels are available then the packet has to wait until the transmission channel is available which leads toward the packet drop increment. With the assumption of 50\% increment in channel availability can improve the network performance.

The results depicted in Fig. 5 and Fig. 6. analyzed with the single-path routing algorithm (SAR-1), Opportunistic Cognitive Routing (OCR), SAAR, and proposed MCSAR. The proposed algorithm achieve significantly better performance than SAAR alogrithm. It is observed that the performance of MCSAR is affected by channel availability and channel conditions. In this paper, we evaluated its improvement for routing in $\mathrm{CCH}$, it makes routing more robust. Therefore, the packet delivery rate is enhanced and the end-to-end delay is decreased concurrently. A comparative study is presented in Fig. 5 in terms of packet delivery improvement where the average packet delivery performance is obtained as 82.5, 77.5, 58.75 and 72.25 by using MCSAR proposed approach, SAAR, Unicast Routing (SAR-1) and Opportunistic Cognitive Routing (OCR). This illustration shows the packet delivery performance depends on the channel availability. The proposed approach achieves a significant performance enhancement as $6.06 \%, 12.42 \%$ and $28.78 \%$ when compared with SAAR, Opportunistic Cognitive Routing (OCR) adnd SAR-1.

With the help of same configuration used for achieving the performance of End-to-End delay is depicted in Fig. 6. The increment in channel availability reduces packet queuing time which leads towards the delay reduction for data transmission through the optimal path. This performance measurement of delay can be reduced by considering proposed approach because the obtained overall delay values are 238.75, 264.25, 342.5, and 300 using MCSAR, SAAR, Unicast Routing (SAR-1), Opportunistic Cognitive Routing (OCR). Since the proposed approach of anypath routing selects the shortest path which reduces time required for packet forwarding. Finally, the overall delay from source to destination node packet transmission is reduced.

In the next phase of performance analysis, we consider varied number of CR routers. Based on this, the communication overhead and computation overhead performance is also computed shown in Fig.7 and Fig. 8. It is easy to understand that the number of routers also affect the network performance, where more number of users leads to more communication overhead. In Fig. 7, we consider a scenario of $400 \mathrm{CR}$ routers in which communication overhead is measured. The data rate follows CBR data traffic where data packets are arriving contineously. This CBR data and channel availability causes congestion and communication overhead. However, as the number of CR routers and channel availability increases, the communication overhead also increases. Furthermore, more number of CR routers can be beneficial for obtaining most suitable path with multiple choices of choosing the shortest path which can be sorted later using improved Bellman-Ford algorithm. The obtained average performance is $362.85 \mathrm{~ms}$, 408.57ms, 515.71ms and 472.14ms using MCSAR, SAAR, Unicast Routing (SAR-1) and 


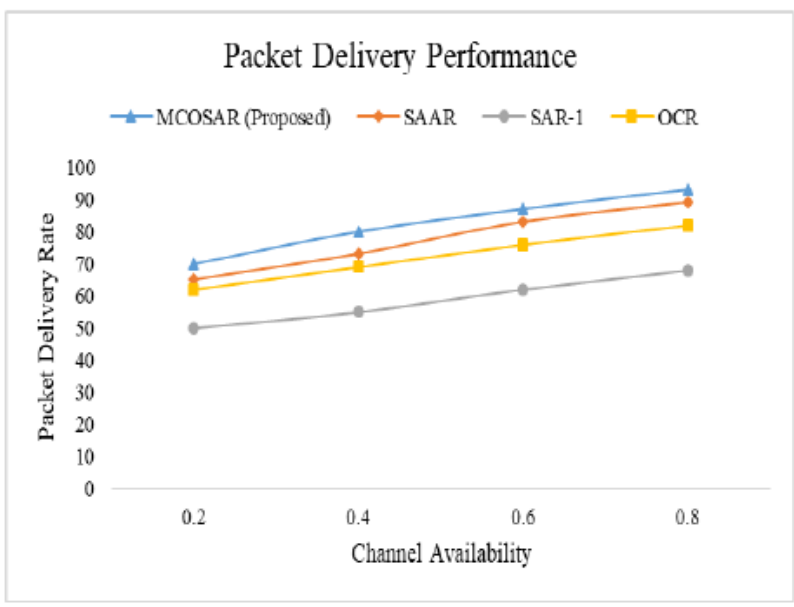

Fig. 5: Packet Delivery Ratio

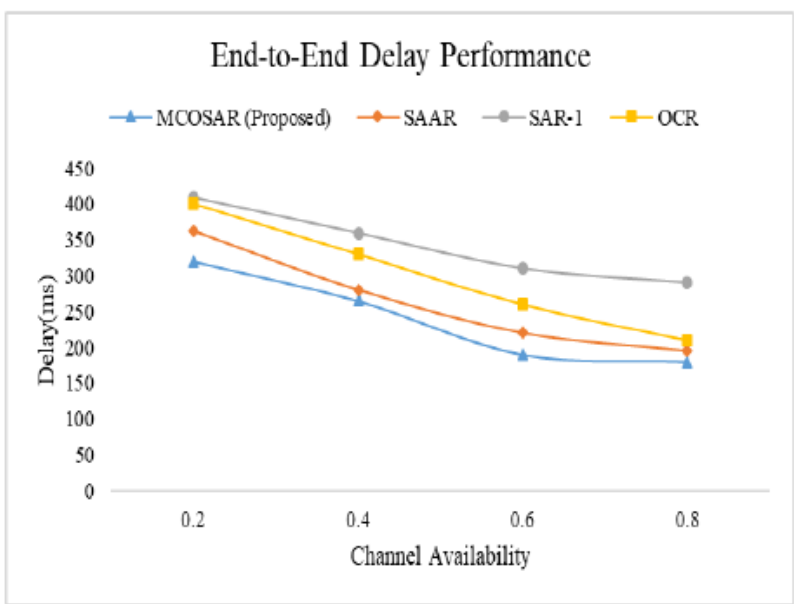

Fig. 6: End-to-End Delay

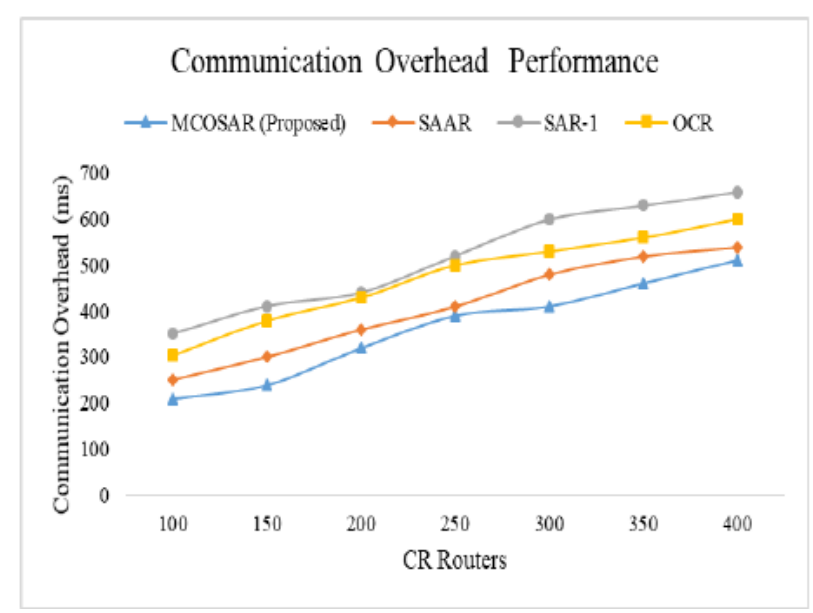

Fig. 7: Communication Overhead 


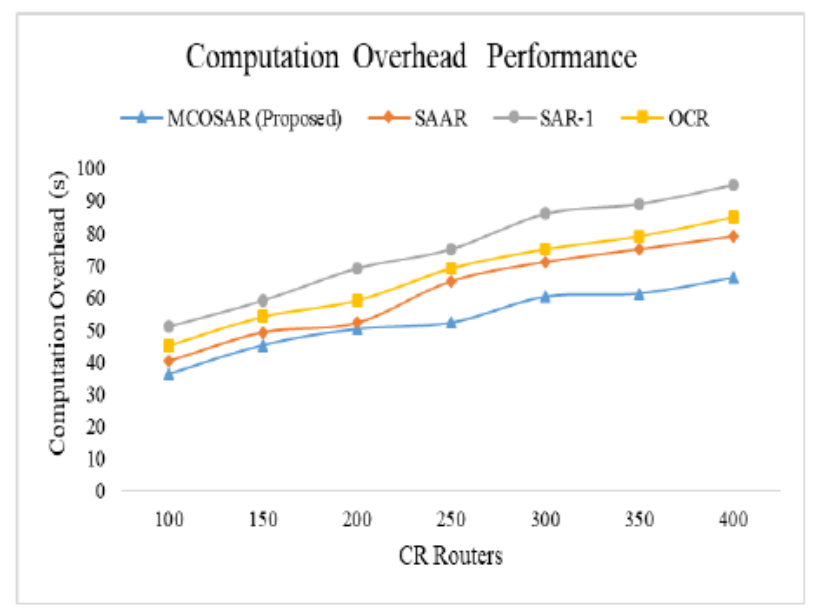

Fig. 8: Computation Overhead

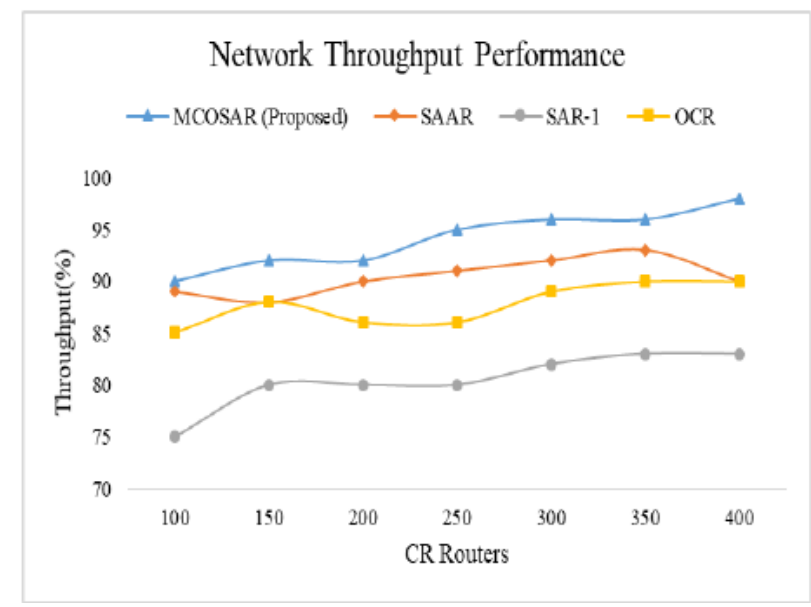

Fig. 9: Network Throughput

Opportunistic Cognitive Routing (OCR). The communication overhead of MCSAR growth takes place as the number of CR routers addition. Besides, the capacity of the statistical data packet and routing table packet in MCSAR are almost identical to SAAR, the communication overhead of MCSAR and SAAR is approaximately identical.

By considering the same communication overhead performance setup for computation overhead, the obtained values using MCSAR, SAAR, Unicast Routing (SAR-1) and Opportunistic Cognitive Routing (OCR) is 52.8s, 61.52s, 74.85s and 66.5s. In Fig. 8 the computation overhead of both MCSAR and SAAR linearly increases as the number of CR routers grows. It is also observed that the computation overhead of MCSAR is diminished remarkably in comparision with SAAR and more appropriate for practical development. In the default set up listed in Table. 2, computation overhead of MCSAR is 52.8s, along the overhead for SAAR is 61.52s. In MCSAR, the routing table requires recomputation during the channel and link statistics updates. Similarly, the increased number of routers and channel availability helps to improve the network performance because availability of more number of channel improves the packet delivery rate and decreases delay to deliver the packet. This can help to enhance the network performance in terms of network throughput. However, the changes in routing table of MCSAR takes place irregularly, over all communication and computation overhead of MCSAR is managable.

Fig. 9 shows the throughput of various algorithms while the maximum size of forwarding sets diminishes from 5 to 2 . Furthermore, it is observed that the throughput of MCSAR occurs no change even the maximum size of forwarding sets updates from 5 to 4 , and it starts to decrease during the maximum size is lesser than 4. However, due to the fact that MCSAR always chooses the nodes with maximum forwarding capability and include them into the forwarding set first. As more number nodes are added into the forwarding set, their achievement on the throughput enhancement of MCSAR is reduced. Therefore, the performance of MCSAR is primarily tested by a less number nodes. Based on the varied number of CR routers, we compute the system throughput performance. The overall average throughput performance of MCSAR, SAAR, Unicast Routing (SAR-1) and Opportunistic Cognitive Routing (OCR) is obtained as 94.14, 90.42, 80.42, and 87.71. 


\section{CONCLUSIONS}

To achieve the efficiency of multi-hop CRNs and make it a powerful spectrum availability by taking into account Cognitive capacity Harvesting Network (CCHN) as an illustration. Cognitive Radio Networks are considered as a promising technique for current wireless communication systems which are helpful for spectrum management. In this paper, we present a novel routing approach for cognitive radio networks to address the spectrum management issue. The proposed routing protocol Multi-Coefficient based Shortest Anypath Routing (MCSAR) is modeled based on the link quality, energy consumption and trust values for identifying the next-hop. Additionally, we incorporate the improved Bellman-Ford algorithm for further optimization of the communication. The proposed routing protocol achieves significant performance in terms of packet delivery ratio, end-to-end delay, communication overhead, computation overhead and throughput compared against conventional/existing anypath routing schemes.

In future work, this approach can be extended to provide the security and uncertain channel usage based issues can also be addressed.

\section{REFERENCES}

[1] E. FCC, "Docket No 03-237 Notice of inquiry and notice of proposed Rulemaking, November 2003," ET Docket, no. 03-237.

[2] F. F. Qureshi, R. Iqbal, and M. N. Asghar, "Energy Efficient Wireless Communication Technique based on Cognitive Radio for Internet of Things," Journal of Network and Computer Applications, vol. 89, pp. 14-25, 2017.

[3] M. Pan, C. Zhang, P. Li, and Y. Fang, "Spectrum Harvesting and Sharing in Multi-Hop CRNs under Uncertain Spectrum Supply," IEEE Journal on Selected Areas in Communications, vol. 30, no. 2, pp. 369-378, 2012.

[4] M. Pan, H. Yue, C. Zhang, and Y. Fang, "Path Selection under Budget Constraints in Multihop Cognitive Radio Networks," IEEE Transactions on Mobile Computing, vol. 12, no. 6, pp. 1133-1145, 2013.

[5] H. Yue, M. Pan, Y. Fang, and S. Glisic, "Spectrum and Energy Efficient Relay Station Placement in Cognitive Radio Networks," IEEE Journal on Selected Areas in Communications, vol. 31, no. 5, pp. 883-893, 2013.

[6] S. Ping, A. Aijaz, O. Holland, and A.-H. Aghvami, "SACRP: A Spectrum Aggregation-based Cooperative Routing Protocol for Cognitive Radio Ad-Hoc Networks," IEEE transactions on communications, vol. 63, no. 6, 2015.

[7] A. Guirguis, M. Karmoose, K. Habak, M. El-Nainay, and M. Youssef, "Cooperation-based Multi-Hop Routing Protocol for Cognitive Radio Networks," Journal of Network and Computer Applications, vol. 110, pp. 27-42, 2018.

[8] H. A. B. Salameh and R. El-Khatib, "Spectrum-Aware Routing in Full-Duplex Cognitive Radio Networks: An Optimization Framework," IEEE Systems Journal, 2018.

[9] Y. B. Zikria, F. Ishmanov, M. K. Afzal, S. W. Kim, S. Y. Nam, and H. Yu, "Opportunistic Channel Selection MAC Protocol for Cognitive Radio Ad Hoc Sensor Networks in the Internet Of Things," Sustainable Computing: Informatics and Systems, vol. 18, pp. 112-120, 2018.

[10] J. Wang, H. Yue, L. Hai, and Y. Fang, "Spectrum-Aware Anypath Routing in Multi-Hop Cognitive Radio Networks," IEEE Transactions on Mobile Computing, vol. 16, no. 4, pp. 1176-1187, 2017.

[11] Y. Liu, L. X. Cai, and X. S. Shen, "Spectrum-Aware Opportunistic Routing in Multi-Hop Cognitive Radio Networks," IEEE Journal on Selected Areas in Communications, vol. 30, no. 10, pp. 1958-1968, 2012.

[12] Y. Liu, L. X. Cai, and X. Shen, "Joint Channel Selection and Opportunistic Forwarding in Multi-Hop Cognitive Radio Networks," in Global Telecommunications Conference (GLOBECOM 2011), 2011 IEEE. IEEE, 2011, pp. 1-5.

[13] Y. Liu, L. X. Cai, X. Shen, and J. W. Mark, "Exploiting Heterogeneity Wireless Channels for Opportunistic Routing in Dynamic Spectrum Access Networks," in Communications (ICC), 2011 IEEE International Conference on. IEEE, 2011, pp. 1-5.

[14] L. Cai, Y. Liu, X. Shen, J. Mark, and D. Zhao, "QoS-Aware Cognitive MAC for Multimedia over Cognitive Radio Networks," Proc. IEEE Globecom10, 2010.

[15] X. Guan, A. Li, Z. Cai, and T. Ohtsuki, "Coalition Graph Game for Robust Routing in Cooperative Cognitive Radio Networks," Mobile Networks and Applications, vol. 20, no. 2, pp. 147-156, 2015.

[16] X. Liu, R. Zhu, B. Jalaian, and Y. Sun, "Dynamic Spectrum Access Algorithm based on Game Theory in Cognitive Radio Networks," Mobile Networks and Applications, vol. 20, no. 6, pp. 817-827, 2015.

[17] H. Fang, L. Xu, J. Li, and K.-K. R. Choo, "An Adaptive Trust-Stackelberg Game Model for Security and Energy Efficiency in Dynamic Cognitive Radio Networks," Computer Communications, vol. 105, pp. 124-132, 2017.

[18] W. Wang, A. Kwasinski, D. Niyato, and Z. Han, "Learning for Robust Routing based on Stochastic Game in Cognitive Radio Networks," IEEE Trans. Commun., PP, vol. 1, 2018.

[19] S. G. K. N., S. H. Manjula, and V. K. R., "A Comprehensive Review on Spectrum Management, Security and Energy-Efficient Cognitive Radio Networks," International Journal of Computer Applications, vol. 182, no. 37, pp. 25-44, 2019.

[20] J. S. P. Singh and M. K. Rai, "CROP: Cognitive Radio Routing Protocol for Link Quality Channel Diverse Cognitive Networks," Journal of Network and Computer Applications, vol. 104, pp. 48-60, 2018.

[21] S. Ji, M. Yan, R. Beyah, and Z. Cai, "Semi-Structure Routing and Analytical Frameworks for Cognitive Radio Networks," IEEE Transactions on Mobile Computing, vol. 15, no. 4, pp. 996-1008, 2016.

[22] K. Aberer, G. Alonso, G. Barrenetxea, J. Beutel, J. Bovay, H. Dubois-Ferrière, D. Kossmann, M. Parlange, L. Thiele, and M. Vetterli, "Infrastructures for a Smart Earth-The Swiss NCCR-MICS initiative-," Praxis der Informationsverarbeitung und Kommunikation, vol. 30, no. 1, pp. $20-25,2007$.

[23] C.-M. Chao, H.-Y. Fu, and L.-R. Zhang, "An Anypath Routing Protocol for Multi-Hop Cognitive Radio Ad Hoc Networks," in Ubiquitous Intelligence and Computing, 2014 IEEE 11th Intl Conf on and IEEE 11th Intl Conf on and Autonomic and Trusted Computing, and IEEE 14th Intl Conf on Scalable Computing and Communications and Its Associated Workshops (UTC-ATC-ScalCom). IEEE, 2014, pp. 127-133.

[24] M. J. Bannister and D. Eppstein, "Randomized Speedup Of The Bellman-Ford Algorithm," in 2012 Proceedings of the Ninth Workshop on Analytic Algorithmics and Combinatorics (ANALCO). SIAM, 2012, pp. 41-47.

[25] X. Fang, D. Yang, and G. Xue, "MAP: Multiconstrained Anypath Routing in Wireless Mesh Networks," IEEE Transactions on Mobile Computing, vol. 12, no. 10, pp. 1893-1906, 2013.

[26] S. Jain and S. R. Das, "Exploiting Path Diversity in the Link Layer in Wireless Ad Hoc Networks," Ad Hoc Networks, vol. 6, no. 5, pp. 805-825, 2008. 